\title{
The Alternative Respiratory Pathway of the Yeast Candida parapsilosis: Oxidation of Exogenous NAD(P)H
}

\author{
By N. M. CAMOUGRAND, A. CHEYROU, M. F. HENRY AND \\ M. G. GUERIN* \\ Institut de Biochimie Cellulaire et de Neurochimie du CNRS, I nue Camille St Saens, \\ 33077 Bordeaux Cedex, France
}

(Received 22 March 1988; revised 9 August 1988)

\begin{abstract}
The yeast Candida parapsilosis possesses two routes of electron transfer from exogenous NAD(P)H to oxygen. Electrons are transferred either to the classical cytochrome pathway at the level of ubiquinone through an NAD(P)H dehydrogenase, or to an alternative pathway at the level of cytochrome $c$ through another NAD(P)H dehydrogenase which is insensitive to antimycin A. Analyses of mitoplasts obtained by digitonin/osmotic shock treatment of mitochondria purified on a sucrose gradient indicated that the NADH and NADPH dehydrogenases serving the alternative route were located on the mitochondrial inner membrane. The dehydrogenases could be differentiated by their $\mathrm{pH}$ optima and their sensitivity to amytal, butanedione and mersalyl. No transhydrogenase activity occurred between the dehydrogenases, although NADH oxidation was inhibited by NADP+ and butanedione. Studies of the effect of NADP+ on NADH oxidation showed that the NADH : ubiquinone oxidoreductase had Michaelis-Menten kinetics and was inhibited by NADP+, whereas the alternative NADH dehydrogenase had allosteric properties (NADH is a negative effector and is displaced from its regulatory site by $\mathrm{NAD}^{+}$or $\mathrm{NADP}^{+}$).
\end{abstract}

\section{INTRODUCTION}

The yeast Candida parapsilosis CBS 7154 was previously described as being strictly dependent on oxidative metabolism for growth (Guérin et al., 1982; Camougrand et al., 1983). It is, nevertheless, able to grow on high concentrations of glucose and also on a glycerol medium supplemented with antimycin A or with drugs acting at the level of mitoribosomal protein synthesis (Guérin et al., 1982; Camougrand et al., 1983, 1986). Besides its normal respiratory pathway, $C$. parapsilosis develops an alternative electron transfer chain which allows the oxidation of exogenous NADH through a second route different from that already described for Saccharomyces cerevisiae (Ohnishi, 1970) or Candida utilis (Bruinenberg et al., 1985). Indeed, in contrast to mammalian mitochondria, which are not permeable to nicotinamide adenine dinucleotides, yeast mitochondria, like plant mitochondria, are able to oxidize exogenous NADH using a second NADH :ubiquinone oxidoreductase located on the outer face of the inner membrane. This pathway is insensitive to rotenone or amytal and is completely inhibited by antimycin A (Ohnishi, 1970). C. utilis also contains a third NADH dehydrogenase, located in the outer membrane, which catalyses the antimycin A-insensitive and piericidin-insensitive oxidation of NADH (Ohnishi et al., 1966). Such an NADH dehydrogenase was also observed in a mutant of $S$. cerevisiae, devoid of ubiquinone, which catalysed electron transfer from NADH to oxidase, using cytochrome $c$ to shuttle electrons between the outer and the inner membrane (De Santis \& Melandri 1984).

Abbreviations: CCCP, carbonyl cyanide m-chlorophenylhydrazone; DCCD, $N, N^{\prime}$-dicyclohexylcarbodiimide; SHAM, salicylhydroxamic acid. 
The alternative pathway of $C$. parapsilosis involves an amytal-sensitive, antimycin-Ainsensitive NADH dehydrogenase that is accessible to external NADH and interacts with the main respiratory chain at the cytochrome $c$ level. Electrons are then transferred either to cytochrome $a a_{3}$ or to the alternative oxidase, a cytochrome absorbing at $590 \mathrm{~nm}$ that is sensitive to salicylhydroxamates and to high concentrations of cyanide (Guérin \& Camougrand, 1986). The sites of action of the inhibitors are shown in Fig. 5 (see Discussion).

It was previously shown (Caubet $e$ al., 1988) that glycolytic pathway activities were rather weak in C. parapsilosis, whereas some enzymes of gluconeogenesis were very active and few were sensitive to catabolite repression. Glucose-6-phosphate dehydrogenase and 6-phosphogluconate dehydrogenase, two enzymes of the hexose monophosphate pathway, were also very active and thus produced NADPH at high rates.

These data led us to investigate whether mitochondria from C. parapsilosis, like those of other yeasts (Djavadi et al., 1980; Bruinenberg et al., 1985), were able to reoxidize exogenous NADPH and, if so, which electron transfer pathway(s) were involved.

Some preliminary results have already been published (Guérin et al., 1987).

\section{METHODS}

Strain and culture. C. parapsilosis CBS 7154 was isolated in this laboratory (Camougrand et al., 1988). Cells were grown at $28^{\circ} \mathrm{C}$ under vigorous aeration on a complete medium supplemented with $2 \%(\mathrm{w} / \mathrm{v})$ glycerol plus or minus (normal cells) antimycin $\mathbf{A}\left(2 \mathrm{mg} \mathrm{ml}^{-1}\right)$.

Preparation and purification of mitochondria. Mitochondria were prepared after digestion of the yeast cell wall with snail (Helix pomatia) juice enzyme (Guérin et al., 1979). Mitochondria were frozen in liquid nitrogen as small pellets (protein concentration 30 to $50 \mathrm{mg} \mathrm{m}^{-1}$ ) and kept at $-80^{\circ} \mathrm{C}$ until use. When required, mitochondria were loaded on to a discontinuous sucrose gradient $(55 \%, 40 \%$ and $30 \%, w / v$, sucrose respectively) or on top of a Percoll solution $(25 \%, \mathrm{w} / \mathrm{v}$, in $0.76 \mathrm{M}$-mannitol $(0.6 \mathrm{M}$ final), $10 \mathrm{mM}$-Tris/maleate, $1 \mathrm{~mm}$-EGTA buffer, pH 6.8, and were centrifuged at $135000 \mathrm{~g}$ for $15 \mathrm{~min}$ in a Beckman TL-100 ultracentrifuge. A $50 \%$ yield of purified mitochondria was generally obtained.

Mitoplasts were prepared using a digitonin treatment (Greenawalt, 1974). Prior to use, digitonin was recrystallized and resuspended in the presence of bovine serum albumin in buffer (respiration medium) that was nearly boiling, and then gently shaken until completely dissolved.

Oxygen uptake was followed polarographically at $28^{\circ} \mathrm{C}$ with a Clark electrode in a medium containing $0.65 \mathrm{M}$ mannitol, $0.36 \mathrm{~mm}-\mathrm{EGTA}, 0.3 \%(\mathrm{w} / \mathrm{v})$ bovine serum albumin and $10 \mathrm{mM}$-Tris/maleate buffer, pH $5.3 \mathrm{or} 6.7$ (respiration buffer).

Enzyme assays. NADH :cytochrome $c$ oxidoreductase (EC 1.6.2.1) and NADPH :cytochrome $c$ oxidoreductase (EC 1.6.2.3) activities were measured by following the reduction of cytochrome $c(550-540 \mathrm{~nm})$; $\Delta \varepsilon=18 \mathrm{~mm}^{-1} \mathrm{~cm}^{-1}$. NADH : ferricyanide oxidoreductase activity was measured by following the reduction of ferricyanide (405-436 nm); $\Delta \varepsilon=0.21 \mathrm{mM}^{-1} \mathrm{~cm}^{-1}$.

Transhydrogenase (EC 1.1.6.1) activity was measured according to the method of Rydstrom (1979), the disappearance of NADP+ and the appearance of $\mathrm{NAD}^{+}$being followed by linking to isocitrate dehydrogenase and alcohol dehydrogenase activities respectively.

Kynurenine hydroxylase (EC 1.99.1.5) was measured according to the method of Hayaishi (1962) at 340$375 \mathrm{~nm}$ in the presence of $10 \mathrm{mM}-\mathrm{KCN}$. The disappearance of NADPH was followed in the absence of the substrate and after addition of DL-kynurenine.

Adenylate kinase (EC 2.7.4.3) was assayed according to Schnaitman \& Greenawalt (1968) at $334 \mathrm{~nm}$ in the absence or presence of $0.1 \%(w / v)$ Triton X-100.

Fumarase (EC 4.2.1.2) was determined according to the method of Massey (1955) at $300-350 \mathrm{~nm}$ by following the disappearance of fumarate at $300 \mathrm{~nm}$.

Protein was determined by the biuret method using bovine serum albumin (Sigma) as standard.

Inhibitor experiments. The stock butanedione solution was prepared by dissolving $25.8 \mu$ butanedione in $1 \mathrm{ml}$ $50 \mathrm{mM}$-Tris/borate buffer, $\mathrm{pH} 7.5$ (the final concentration of inhibitor was $0.3 \mathrm{M}$ ). Other inhibitors were dissolved in double-distilled water (amytal, KCN) or in methanol (antimycin A, SHAM).

Chemicals. NAD(P)H, NAD ${ }^{+}, \mathrm{NADP}^{+}$, antimycin A, digitonin and cytochrome $c$ were purchased from Sigma; diacetyl 2,3-butanedione was from Aldrich; DL-kynurenine was from Fluka; SHAM was from EGA; amytal was from Serva and KCN from Merck.

\section{RESULTS}

Oxidation of exogenous NADH and NADPH is a general property of mitochondria from plants and fungi (Schuurmans-Steckhoven, 1966; Schwitzguebel \& Palmer, 1981; Palmer \& 


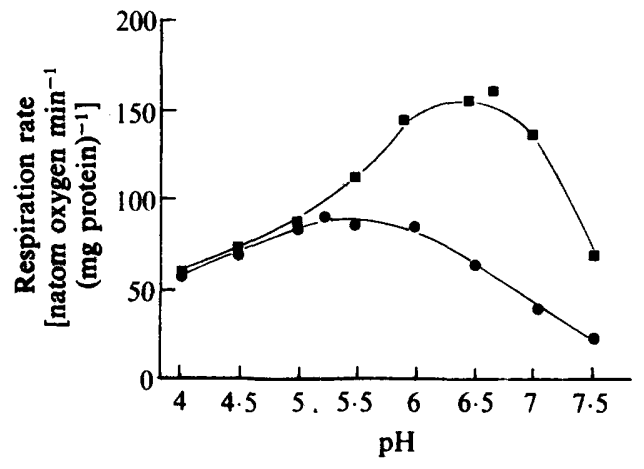

Fig. 1. Determination of optimum pH for NADH and NADPH oxidation. Mitochondria from normal cells (1 mg protein) were suspended in $3 \mathrm{ml}$ of respiration buffer at different $\mathrm{pH}$ values and oxygen uptake was measured. $\mathrm{a}, 3 \mathrm{mM-NADH}$;, $3 \mathrm{~mm}$-NADPH.

Table 1. NAD $(P) H$ :cytochrome c oxidoreductase activities of mitochondria from $C$. parapsilosis

Mitochondria from normal cells were purified (gradient mitochondria) or not (crude mitochondria) on a Percoll gradient. NAD(P)H :cytochrome $c$ oxidoreductase activities were measured at pH 6.7 and 5.3 respectively in respiration buffer at $20^{\circ} \mathrm{C}$. Results given are means of three experiments; the variability was $8 \%$. Numbers in parentheses are percentage inhibition. NM, Not measurable.

\begin{tabular}{|c|c|c|c|c|c|c|}
\hline & \multicolumn{6}{|c|}{ Specific activity $\left[\mu \mathrm{mol} \mathrm{min} \mathrm{m}^{-1}(\mathrm{mg} \text { protein })^{-1}\right]$} \\
\hline & \multicolumn{3}{|c|}{ Crude mitochondria } & \multicolumn{3}{|c|}{ Gradient mitochondria } \\
\hline & \multirow{2}{*}{$\begin{array}{l}\text { NADH } \\
1 \mathrm{mM}\end{array}$} & \multicolumn{2}{|c|}{ NADPH } & \multirow{2}{*}{$\begin{array}{l}\text { NADH } \\
1 \mathrm{mM}\end{array}$} & \multicolumn{2}{|c|}{ NADPH } \\
\hline & & $50 \mu \mathrm{M}$ & $3 \mathrm{~mm}$ & & $50 \mu \mathrm{M}$ & $3 \mathrm{~mm}$ \\
\hline $\begin{array}{l}\text { Control } \\
\text { Amytal (2 mM) } \\
\left.\text { Antimycin A (1 } \mu \mathrm{g} \mathrm{ml}^{-1}\right)\end{array}$ & $\begin{array}{l}90 \\
50(44 \%) \\
17(81 \%)\end{array}$ & $\begin{array}{l}18 \\
19(0 \%) \\
18(0 \%)\end{array}$ & $\begin{array}{l}60 \\
45(25 \%) \\
55(8 \%)\end{array}$ & $\begin{array}{l}92 \\
37(60 \%) \\
10(89 \%)\end{array}$ & $\begin{array}{l}\text { NM } \\
\text { MM } \\
\text { NM }\end{array}$ & $\begin{array}{l}28 \\
14(50 \%) \\
12(57 \%)\end{array}$ \\
\hline
\end{tabular}

Møller, 1982), the two dehydrogenase activities being distinguished by their optimal pH values and their sensitivities to various inhibitors. The optimum $\mathrm{pH}$ for the reaction varies with the origin of the mitochondria; for instance, in mitochondria isolated from Jerusalem artichoke tubers, the optimum pH values were 7.0 and 6.0 for NADH and NADPH respectively, whereas they were 7.1 and 6.6 respectively in mitochondria from Arum maculatum spadices (Møller \& Palmer, 1981 a) and 7 and 7.5 respectively in mitochondria from Neurospora crassa (Møller et al., 1982). Similarly, the oxidation of exogenous NADPH by plant mitochondria is sensitive to amytal (Koeppe \& Miller, 1972), whereas the oxidation of NADH is not. Measurements of the rates of NADH and NADPH oxidation by $C$. parapsilosis CBS 7154 mitochondria at different pH values (Fig. 1) showed that these activities were maximal at pH 6.8 and 5.3 respectively.

$\mathrm{NAD}(\mathrm{P}) \mathrm{H}$ : cytochrome $c$ oxidoreductase activities were measured at these optimal values. A single kinetic system was observed for NADH : cytochrome $c$ oxidoreductase activity $\left(K_{\mathrm{m}}=3 \pm 2 \mu \mathrm{M}\right)$, whereas two were observed for NADPH :cytochrome $c$ oxidoreductase activity: one with a high affinity for NADPH $\left(K_{\mathrm{m}}=10 \pm 7 \mu \mathrm{M}\right)$ and the other with a low affinity $\left(K_{\mathrm{m}}=817 \pm 200 \mu \mathrm{M}\right)$. The use of amytal and antimycin A as inhibitors of NADPH oxidation indicated that the high affinity system was amytal-insensitive and the low affinity system was amytal-sensitive and, to a lesser extent, antimycin-A-sensitive (Table 1). The former system most probably resulted from microsomal contamination of the mitochondrial fraction. Indeed, purification of mitochondria (as seen from electron micrographs: not shown) on a Percoll gradient led to the loss of the high affinity system (Table 1). It must be noted that the purified mitochondrial fraction always retained the amytal-sensitive NADPH:cytochrome $c$ oxido- 
Table 2. Comparative enzyme activities of mitochondria and mitoplasts from

$$
\text { C. parapsilosis }
$$

Mitochondria from normal cells were purified on a sucrose gradient and then mitoplasts were obtained following a digitonin-osmotic shock treatment. Enzyme activities were measured as described in Methods. The values represent total activity. NM, Not measurable.

\begin{tabular}{|c|c|c|c|c|}
\hline & $\begin{array}{l}\text { Protein } \\
\text { (mg) }\end{array}$ & $\begin{array}{l}\text { Adenylate kinase } \\
\left(\text { (nmol } \text { min }^{-1}\right)\end{array}$ & $\begin{array}{c}\text { Kynurenine } \\
\text { hydroxylase } \\
(\text { nmol min }\end{array}$ & $\begin{array}{l}\text { Fumarase } \\
\text { (Units)* }\end{array}$ \\
\hline Mitochondria & 60 & 12000 & 6650 & 160 \\
\hline Mitoplasts & $15 \cdot 5$ & 800 & 200 & NM \\
\hline Supernatant & 42 & 13500 & 4450 & 4 \\
\hline
\end{tabular}

* One Unit is equivalent to a $\Delta A$ of $0.01 \mathrm{~min}^{-1}$.

reductase activity. From Table 1 it can be seen that for NADPH :cytochrome $c$ oxidoreduction there was a net increase in sensitivity to inhibitors which paralleled the decrease in specific activity. In contrast, NADH : cytochrome $c$ oxidoreductase exhibited only a slight increase in sensitivity to inhibitors. The fact that the specific activity did not increase after purification was due to the Percoll gradient since, after purification on a sucrose gradient, specific activity increased by $20 \%$.

\section{Localization of NADH and NADPH dehydrogenases within the mitochondria}

In an attempt to localize these dehydrogenases on either the inner or outer membrane of the mitochondria, mitoplasts were prepared as described in Methods. Activities of marker enzymes were determined in the different fractions, i.e. kynurenine hydroxylase for the outer membrane, adenylate kinase as indicative of the intermembrane space, NAD(P)H:ferricyanide oxidoreductases in the presence or absence of Triton X-100 for outer-facing and internally located dehydrogenases respectively, since ferricyanide does not permeate the membrane (Von Jagow and Klingenberg, 1970), and fumarase for the matrix. Absorption difference spectra and oxygen uptakes were recorded concomitantly.

A 15 min digitonin treatment $\left.[0.5 \mathrm{mg} \text { (mg protein })^{-1}\right]$ at $0{ }^{\circ} \mathrm{C}$ or a 15 min osmotic shock $(0.35$ M-mannitol) resulted in a loss of approximately $50 \%$ of the kynurenine hydroxylase and NAD(P)H oxidases activities. No real improvement could be obtained with the same treatment at room temperature. Combination of both treatments, resulted in the disappearance of kynurenine hydroxylase and adenylate kinase activities from the mitoplast pellet, both activities being recovered in the supernatant fraction (Table 2). Results in Table 3 show that although marker enzymes for outer membrane and intermembrane space were lost after detergent treatment, the inner membrane retained its integrity since NAD(P)H :ferricyanide oxidoreductase activities increased after detergent addition; moreover both activities were sensitive to amytal.

Measurements of NADH and NADPH dehydrogenase activities were done on mitochondria and mitoplasts isolated from cells grown in the presence or absence of antimycin $\mathrm{A}$, since the latter growth conditions enhanced the alternative pathway. It can be seen from Table 3 that whatever the culture conditions, mitoplasts retained their relative cyanide-insensitivity. The weak respiratory rate of mitoplasts is due to the loss of cytochrome $c$ during their preparation (measured by spectrophotometry); addition of cytochrome $c$ enhanced the respiratory rate, (which remained partially cyanide resistant) by $300 \%$ (not shown).

\section{Is there a transhydrogenase?}

Based on the activities of the NADH and NADPH oxidase systems measured from oxygen consumption, the rate of NADPH oxidation was about $65 \%$ of that of NADH (Table 3); both NADH and NADPH oxidations were coupled to phosphorylation, but respiratory control was lower with NADPH than with NADH (respiratory control ratios $=1.7$ and 2.5 respectively). Comparison of sensitivities to amytal and cyanide (both $0.1 \mathrm{mM}$ ) of NADH and NADPH 


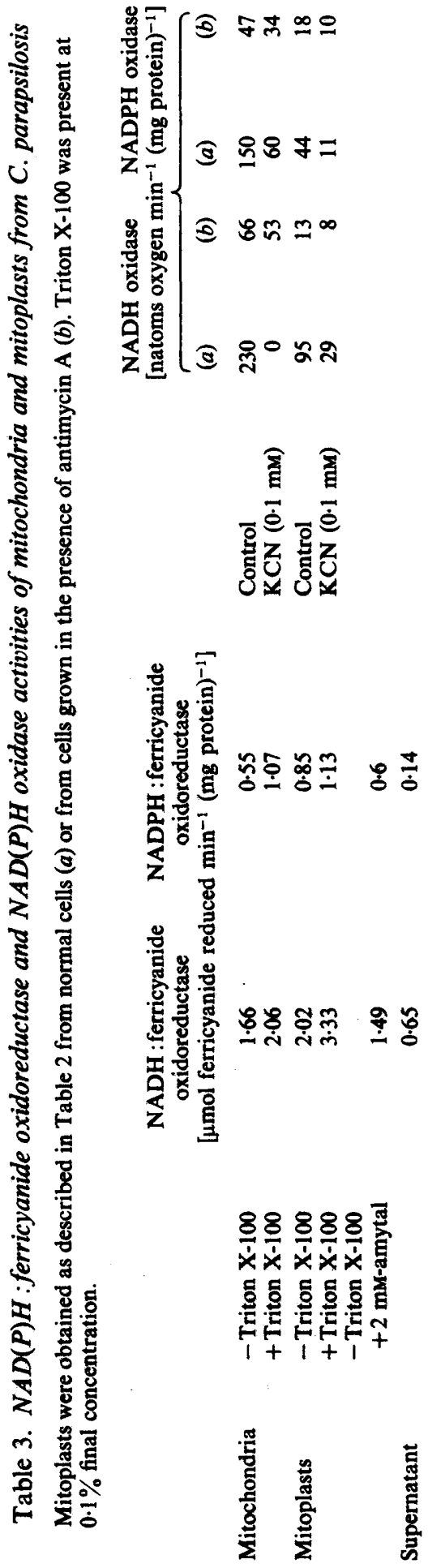




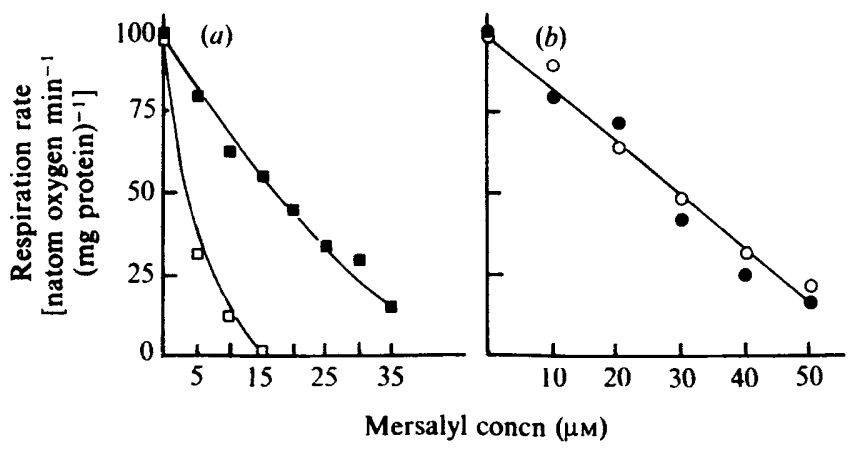

Fig. 2. Effect of mersalyl on NAD(P)H oxidation. Mitochondria from normal cells were purified on a sucrose gradient and pre-incubated with $0.3 \mu \mathrm{g}$ antimycin $A$ in the presence or absence of $10 \mathrm{~mm}$ butanedione. Respiration was induced by addition of $3 \mathrm{mM}-\mathrm{NADH}$ in the presence of different concentrations of mersalyl and initial oxidation rates were measured. $\mathrm{NADH}, \square, \mathrm{NADH}+$ butanedione;, NADPH; O, NADPH + butanedione.

oxidation indicated that respiration with NADPH was more amytal sensitive and cyanide resistant than with NADH, and therefore that NADPH oxidation proceeded mainly through the secondary pathway. However, both oxidations were fully inhibited by the simultaneous addition of cyanide and amytal (not shown).

In view of these differences the problem was to know whether the appearance of the sensitivity to amytal of NADH oxidation was due to a partial oxidation of NADH by NADP+ by a transhydrogenase which catalysed the reversible transfer of hydrogen between NADH and $\mathrm{NADP}^{+}$. Indeed a transhydrogenase system has often been described in animal mitochondria, but very little information is available about the presence of such an enzyme complex in yeast mitochondria. One report indicated that $C$. utilis mitochondria seemed unable to interconvert NADH and NADPH through a transhydrogenation mechanism (Bruinenberg et al., 1985). To test this hypothesis, several experiments were attempted.

First, the effect of increasing concentrations of NADP ${ }^{+}$on the oxidation rate of NADH, at pH 6.8 and 5.3, was assayed. At pH 6.8, successive additions of 2 mM-NADP ${ }^{+}$inhibited NADH oxidation $\left(26 \%\right.$ inhibition for $\left.12 \mathrm{mM}-\mathrm{NADP}^{+}\right)$. When the same experiment was done with NADPH, addition of $\mathrm{NAD}^{+}$had little effect on the NADPH oxidation rate $(4 \%$ inhibition for 12 mM-NAD ${ }^{+}$.

In a second set of experiments, the effect on $\mathrm{NAD}(\mathrm{P}) \mathrm{H}$ oxidation of two inhibitors of transhydrogenase, the carboxyl-group modifier DCCD (Phelps \& Hatefi, 1984) and the arginine-binding reagent butanedione (Riordan, 1973), were examined. DCCD inhibited both NADH and NADPH oxidation; in contrast, butanedione had no effect on NADPH oxidation, but inhibited the oxidation of NADH by 30 to $50 \%$.

Thirdly, the effect of mersalyl, a thiol reagent which was shown to inhibit NADH dehydrogenase more than NADPH dehydrogenase in plant mitochondria (Møller \& Palmer, $1981 b$ ) was investigated. A control experiment showed that mersalyl had no effect on the oxidation of pyruvate + malate, which confirmed that mersalyl did not permeate the mitochondrial membrane (Klingenberg et al., 1974) and/or that it was without effect on the internal NADH dehydrogenase. In a further experiment, NAD(P)H oxidation was measured in the presence of sufficient antimycin A to inhibit the main respiratory chain by $80 \%$. Under these conditions the NADH and NADPH dehydrogenases were $50 \%$ inhibited by $17 \mu \mathrm{M}$ and $30 \mu \mathrm{M}$ mersalyl respectively. However, when mitochondria were pre-incubated with $10 \mathrm{~mm}$ butanedione, the mersalyl sensitivity of NADPH oxidation remained unchanged whereas $50 \%$ inhibition of NADH oxidation was obtained with $3 \mu \mathrm{M}$-mersalyl instead of $17 \mu \mathrm{M}$ (Fig. 2).

Since these results suggested the presence of a transhydrogenase, such an activity was monitored (with NADH and NADP+ as substrate), in the presence of antimycin A, amytal and SHAM to prevent any NADH oxidation. In the absence of mitochondria no transhydrogenation 


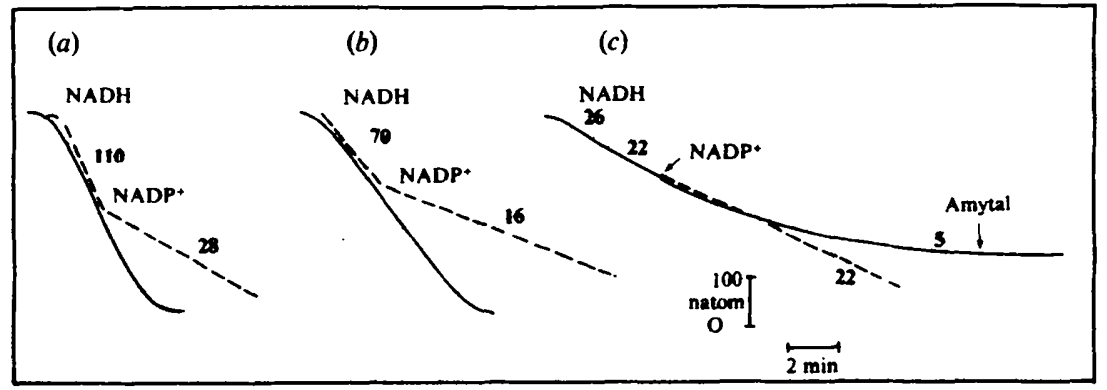

Fig. 3. Effect of NADP+ on NADH oxidation. Mitochondria from normal cells were purified on a sucrose gradient and suspended in respiration buffer, pH 6.8. Respiration was induced with 1 mMNADH; Additions 9 mu-NADP+; 2 mu-amytal. (a) Respiration monitored in the absence of inhibitors. (b) Mitochondria pre-incubated in the presence of 1 mM-SHAM and 2 mu-amytal. (c) Mitochondria pre-incubated in the presence of $0.2 \mu 8$ antimycin A (ms protein) ${ }^{-1}$. Values in boll type are respiration rates [natom oxygen $\mathrm{min}^{-1}$ (mg protein) ${ }^{-1}$ ].

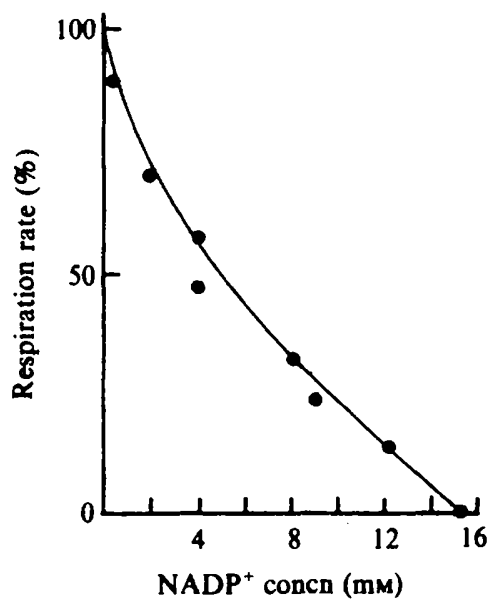

Fig. 4. Inhibition of NADH oxidation by NADP+. Mitochondria from normal cells were purified on a sucroee gradient and suspended in respiration buffer, pH 6.8. Respiration was induced with 1 muNADH and successive additions of NADP+ were made.

occurred within $2 \mathrm{~h}$, whereas in the presence of mitochondria a small amount of NAD ${ }^{+}$ appeared after $1 \mathrm{~h}$ incubation. The level of transhydrogenation never exceeded $16 \%$ even after $2 \mathrm{~h}$ incubation; moreover, this activity was insensitive to butanedione (not shown).

From these results, it was concluded that the effect of NADP+ and butanedione could be better explained by both compounds acting on NADH dehydrogenase rather than by the presence of a transhydrogenase.

We therefore investigated the role of NADP+ on NADH oxidation by mitochondria from cells grown in the presence of antimycin $A$ and purified on a sucrose gradient. The action of $\mathrm{NADP}^{+}$was studied on NADH oxidation, in the presence or absence of respiratory inhibitors. In the absence of inhibitors or in the presence of amytal and SHAM, addition of NADP+ inhibited the respiration rate (Fig. $3 a, b$ ): $50 \%$ inhibition was obtained with 5 mu-NADP+ (Fig. 4). NADH : ubiquinone oxidoreductase exhibited Michaelis-Menten kinetics with a $K_{m}$ for NADH (NADH $\rightarrow \mathrm{O}_{2}$ ) of $50 \mu \mathrm{m}$. In contrast, the alternative NADH dehydrogenase behaved in a different way: in the presence of antimycin A, the rate of NADH oxidation decreased progressively during the experiment, the final respiratory rate falling to only $20 \%$ of the initial 
rate; addition of $\mathrm{NADP}^{+}$did not affect the initial respiratory rate but prevented the subsequent loss of activity (Fig. 3c), indicating that this enzyme is allosteric. To see if inhibition of NADH oxidation was due to the substrate or to the product of the reaction, $\mathrm{NAD}^{+}$was added prior to NADH : NAD ${ }^{+}$had no inhibitory effect and indeed slightly stimulated NADH oxidation (not shown). The same effect was observed with AMP, which is known to stimulate alternative respiration of plants and micro-organisms (Sotthibandhu \& Palmer, 1975; Hanssens and Verachtert, 1976; Doussieres et al., 1979; Vanderleyden et al., 1979).

Similar experiments were also done with NADPH as substrate in the presence of antimycin A. The NADPH dehydrogenase behaved as did the NADH dehydrogenase, i.e. the NADPH oxidation rate was progressively inhibited during the experiment and this inhibition was partially released by the addition of $\mathrm{NADP}^{+}$(not shown).

\section{DISCUSSION}

The results presented above demonstrate that mitochondria from $C$. parapsilosis, like mitochondria from other yeasts (Bruinenberg et al., 1985; Djavadi et al., 1980) support oxidative phosphorylation with exogenous NADH and NADPH. However, in contrast to mitochondria from $C$. utilis (Bruinenberg et al., 1985), both NADPH oxidation and NADH oxidation were only partly antimycin A sensitive. The remaining respiration was inhibited by amytal, which indicated that electon flow from NADPH proceeded through both the classical and the secondary respiratory pathways.

In this paper we attempted to answer three questions. (1) Where are the NAD(P)H dehydrogenases linked to the secondary pathway localized? (2) Are NADPH and NADH oxidized by a common dehydrogenase or by two separate dehydrogenases? (3) Is a transhydrogenase present which is able to interconvert NADH and NADPH?

(1) Intact mitoplasts exhibited both NADH and NADPH oxidation which was partly amytal sensitive, partly cyanide sensitive and inhibited by mersalyl, which does not permeate the inner membrane, thus indicating that NAD(P)H dehydrogenase was located on the outer face of the inner mitochondrial membrane.

(2) Several lines of evidence indicated that the alternative pathway involved dehydrogenases specific for NADH and NADPH respectively, as distinct from the NAD(P)H : ubiquinone oxidoreductase. Firstly, in contrast to plant mitochondria (Koeppe \& Miller, 1972), both NADH and NADPH oxidation were amytal sensitive, but the former less than the latter. Secondly, NADH dehydrogenase, but not NADPH dehydrogenase, is butanedione sensitive. Thirdly, NADH dehydrogenase was more mersalyl sensitive than NADPH dehydrogenase, especially in the presence of butanedione.

(3) The fact that NADH oxidation was inhibited by NADP+ and by butanedione, a specific inhibitor of mammalian transhydrogenase, prompted us to inquire whether a transhydrogenase was present which could interconvert NADH and NADPH. However, the small amount of $\mathrm{NAD}^{+}(16 \%)$ obtained after $2 \mathrm{~h}$ did not support this possibility. It seemed more likely that butanedione, an arginine-binding reagent (Riordan, 1973), either reacted at the active site of the NADH dehydrogenase or modified its conformation; this latter hypothesis could explain the greater mersalyl sensitivity of NADH dehydrogenase in the presence of butanedione.

A schematic representation, summarizing our findings, of the NAD(P)H oxidation pathways, is shown in Fig. 5.

The effect of NADP+ addition on NADH oxidation was further investigated in order to find evidence for an eventual regulatory function of NADP+. First, measurements of NADH oxidation through the ubiquinone :cytochrome $c$ oxidase pathway or through the alternative pathway allowed discrimination between the behaviour of the two enzymes towards their substrates. The former was a Michaelian enzyme inhibited by NADP ${ }^{+}$(Figs $3 b$ and 4), whereas the latter was allosteric. Indeed, the alternative NADH dehydrogenase was regulated by both the reduced and oxidized forms of nicotinamide adenine dinucleotide. Results obtained suggest that on the one hand, NADH was an negative effector and on the other hand that NADP+ ${ }^{+}$and $\mathrm{NAD}^{+}$displace NADH from its negative allosteric site. 


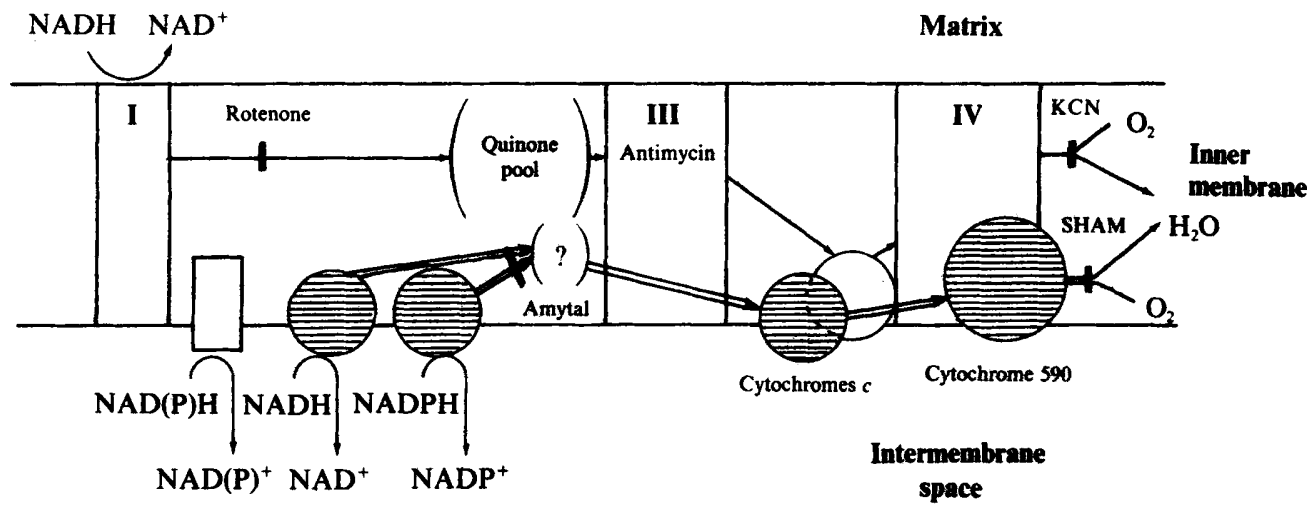

Fig. 5. NAD(P)H oxidation pathways in C. parapsilosis mitochondria.

We postulated that cytoplasmic NADP+ regulates the functioning of both pathways by inhibiting the NADH :ubiquinone oxidoreductase and by modifying the conformation of the alternative NADH dehydrogenase, thus preventing the negative effect of NADH on the enzyme.

Further experiments are underway on the purified enzymes.

The authors wish to thank Dr Jean-Pierre Mazat for the elaboration of the different computer programs. This work was performed with grants from the University of Bordeaux II, the Centre National de la Recherche Scientifique, and the Ministère de la Recherche et de la Technologie (PIRSEM-ATP/AFME 509-810).

\section{REFERENCES}

Bruinenberg, P. M., van Dijken, J. P., Kuenen, J. G. \& SCHEFFERS, W. A. (1985). Oxidation of NADH and NADPH by mitochondria from the yeast Candida utilis. Journal of General Microbiology 131, 1043-1051.

Camougrand, N., Caubet, R. \& Gúkrin, M. (1983). Evidence for an alternative and non-phosphorylating pathway for NADH reoxidation in a yeast resistant to glucose repression. European Journal of Biochemistry 135, 367-371.

Camougrand, N., Velours, G. \& Gúfrin, M. (1986). The resistance of $C$. parapsilosis to drugs. Biology of the Cell 58, 71-78.

Camougrand, N., Mila, B., Velours, G., Lazowska, J. \& GutRIN, M. (1988). Discrimination between different groups of Candida parapsilosis by mitochondrial DNA restriction analysis. Current Genetics 13, 445-449.

Caubet, R., Gufrin, B. \& Gúfrin, M. (1988). Comparative studies on the glycolytic and hexose monophosphate pathways in Candida parapsilosis and Saccharomyces cerevisiae. Archives of Microbiology 149, 324-329.

De Santis, A. \& Melandri, B. A. (1984). The oxidation of external NADH by an intermembrane electron transfer in mitochondria from the ubiquinone-deficient mutant E3-24 of Saccharomyces cerevisiae. Archives of Biochemistry and Biophysics 232, 354-365.

Diavadi, F. H. S., Moradi, M. \& DuavadiOHANIANCE, L. (1980). Direct oxidation of NADPH by submitochondrial particles from $S$. cerevisiae. European Journal of Biochemistry 107, 501-504.
Doussiekre, J., Sainsard-Chanet, A. \& Vignais, P. V. (1979). The respiratory chain of Paramecium tetraurelia in wild type and the mutant C11. II. Cyanide insensitive respiration: function and regulation. Biochimica et biophysica acta 548, 236-252.

Grienawalt, J. W. (1974). The isolation of outer and inner mitochondrial membranes. Methods in Enzymology 31, 310-323.

Gúfrin, M. \& CaMougrand, N. (1986). The alternative oxidase of $C$. parapsilosis. European Journal of Biochemistry 159, 519-524.

Gúfrin, B., Labbe, P. \& SomLo, M. (1979). Preparation of yeast mitochondria (S. cerevisiae) with good $\mathrm{P} / \mathrm{O}$ and respiratory control ratios. Methods in Enzymology 55, 149-159.

Gúbrin, M., Camougrand, N., Velours, G. \& Gutrin, B. (1982). New mutants resistant to glucose repression affected in the regulation of the NADH oxidation. European Journal of Biochemistry 124, 457-463.

Gutrin, M., Camougrand, N., Cheyrou, A. \& HENRY, M. F. (1987). Another type of alternative electron transfer pathway in the yeast Candida parapsilosis. In Plant Mitochondria : Structural, Functional and Physiological Aspects, pp. 243-246. Edited by A. L. Moore \& R. B. Beechey. New York: Plenum Press.

HaNsSENS, L. \& Verachtrert, H. (1976). Adenosine 5'monophosphate-stimulated cyanide insensitive respiration in mitochondria of Moniliella tomentosa. Journal of Bacteriology 125, 829-836.

HAYAISHI, O. (1962). Kynurerine hydroxylase. Methods in Enzymology 5, 807-809. 
Korrpe, D. E. \& Miller, J. (1972). Oxidation of reduced nicotinamide adenine dinucleotide phosphate by isolated corn mitochondria. Plant Physiology 49, 353-357.

KLINGengerg, M., DURAND, R. \& GuERIN, B. (1974). Analysis of the reactivity of SH-reagents with the mitochondrial phosphate carrier. European Journal of Biochemistry 42, 135-150.

MAsseY, V. (1955). Fumarase. Methods in Enzymology 1, 729-733.

Møller, I. M. \& Palmer, J. P. (1981 a). Properties of the oxidation of exogenous NADH and NADPH by plant mitochondria. Evidence against a phosphatase or a mitochondria nucleotide transhydrogenase being responsible for NADPH oxidation. Biochimica et biophysica acta 638, 225-233.

MøLlER, I. M. \& PALMER, J. P. (1981 $b$ ). The inhibition of exogenous NAD(P)H oxidation in plant mitochondria by chelators and mersalyl as a function of pH. Physiologia plantarum 53, 413-420.

Møller, I. M., Schwitzguebel, J. P. \& Palmer, J. M. (1982). Binding and screening by cations and the effect on exogenous NAD(P)H oxidation in Neurospora crassa mitochondria. European Journal of Biochemistry 123, 81-88.

OHNISH, T. (1970). Induction of site I phosphorylation in vivo in Saccharomyces carlsbergensis. Biochemical and Biophysical Research Communications 41, 344 352.

OHNISH, T., SOTtocasa, G. \& ERnster, L. (1966). Current approaches to the mechanism of energycoupling in the respiratory chain. Studies with yeast mitochondria. Bulletin de la Société de chimie biologique 48, 1189-1203.

PALMER, J. M. \& MOLLER, I. M. (1982). Regulation of NAD(P)H dehydrogenase in plant mitochondria. Trends in Biochemical Sciences 7, 258-261.
Phelps, D. C. \& HatefI, Y. (1984). Interaction of purified nicotinamide nucleotide transhydrogenase with dicyclohexylcarbodiimide. Biochemistry 23, 4475-4480.

RioRDAN, J. F. (1973). Functional arginyl residues in carboxypeptidase A. Modification with butanedione. Biochemistry 12, 3915-3923.

RYDSTROM, J. (1979). Assay of nicotinamide nucleotide transhydrogenases in mammalian, bacterial and reconstituted systems. Methods in Enzymology 55, 261-275.

Schnartman, C. A. \& Greenawalt, J. W. (1968). Enzymatic properties of the inner and outer membranes of rat liver mitochondria. Journal of Cellular Biology 38, 158-175.

SChuURMans-STERHoven, F. M. A. H. (1966). Studies on yeast mitochondria. I. Existence of three phosphorylation sites along the respiratory chain of yeast mitochondria. Archives of Biochemistry and Biophysics 115, 555-568.

SChWtrzguebel, J. P. \& Palmer, J. M. (1981). Properties of mitochondria isolated from Neurospora crassa grown with acetate. FEMS Microbiology Letters 11, 273-277.

Sotthibandhu, R. \& Palmer, J. M. (1975). The activation of non-phosphorylating electron transport by adenine nucleotides in Jerusalem artichoke (Helianthus tuberosus) mitochondria. Biochemical Journal 152, 637-645.

VANDERLEYDEN, J. KURTH, I. \& VerachterT, H. (1979). Characterization of cyanide-insensitive respiration in mitochondria and submitochondrial particles in Moniliella tomentosa. Biochemical Journal 182, 437-443.

Von Jagow, G. \& KLINGenberg, M. (1970). Pathways of hydrogen in mitochondria of $S$. carlsbergensis. European Journal of Biochemistry 12, 583-592. 